\title{
LOS PECES FÓSILES DE LA FORMACIÓN MEXCALA (CRETÁCICO SUPERIOR) EN EL ESTADO DE GUERRERO, MÉXICO
}

\author{
JESÚS ALVARADO-ORTEGA \\ Departamento de Zoología, Instituto de Biología, UERJ, São Francisco Xavier 524, Maracanã, 20559-900, \\ Rio de Janeiro, RJ, Brasil. jalvaradoortega@yahoo.com.mx \\ LUIS M. GARIBAY-ROMERO \\ Unidad Académica de Ciencias de la Tierra, UAG, Ex-hacienda de San Juan Bautista S/N, Taxco el Viejo, \\ Guerrero, 40200 México. garibay_luis@hotmail.com \\ ALBERTO BLANCO-PIÑÓN \\ Centro de Investigaciones en Ciencias de la Tierra, UAEH, Hidalgo, México.albblanc@yahoo.com \\ GERARDO GONZÁLEZ-BARBA \\ Departamento de Biología Marina, UABC, Cx. P. 23080, La Paz, BCS, México. gerardo@uabcs.mx \\ FRANCISCO J. VEGA \& ELENA CENTENO-GARCÍA \\ Instituto de Geología, UNAM, Ciudad Universitaria, Coyoacán, DF, 04510 México. \\ vegver@servidor.unam.mx,centeno@servidor.unam.mx
}

\begin{abstract}
RESUMO - Neste trabalho são descritos, pela primeira vez, os peixes fósseis provenientes do Estado de Guerrero, México. O material fóssil foi coletado em seis localidades do Cretáceo Superior (TuronianoMaastrichtiano) pertencentes à Formação Mexcala. Os fósseis representam grupos de condrícties, incluindo dentes de Ptychodus mortoni, P. decurrens, Serratolamna serrata, Squalicorax sp. e Cretoxyrhina cf. mantelli, além de vértebras de lamniformes indeterminados. Os osteícties identificados incluem restos de ichthyodectiformes, Tselfatia sp., Enchodus sp., Pachyrhizodus cf. caninus, dercetídeos, assim como distintos ossos, dentes e escamas de teleósteos indeterminados. Esta contribuição representa o registro mais austral na América do Norte de peixes do Cretáceo Superior, a qual permite ampliar a distribução geográfica dos táxons mencionados, incluindo agora o extremo oeste do Mar de Tétis ou Paleo Golfo de México. Além disso, é registrada a descoberta mais recente (Maastrichtiano) de Ptychodus mortoni.
\end{abstract}

Palavras-chave: Peixes, Formação Mexcala, Cretáceo superior, México.

ABSTRACT - FOSSIL FISHES FROM THE MEXCALA FORMATION (UPPER CRETACEOUS) IN THE GUERRERO STATE, MEXICO. Just discovered fossil fishes from Guerrero State, Mexico, are described. The specimens were collected in six localities from the Upper Cretaceous (Turonian-Maastrichtian) Mexcala Formation and belong to different chondrichthyan groups. The fish remains include teeth of Ptychodus mortoni, P. decurrens, Serratolamna serrata, Squalicorax sp., and Cretoxyrhina cf. mantelli, as well as unidentified lamniform vertebrae. Identified osteichthyans include remains of ichthyodectiforms, Tselfatia sp., Enchodus sp., Pachyrhizodus cf. caninus, dercetids, and several bones, teeth and scales of unidentified taxa. This contribution represents the southern most report of Upper Cretaceous fishes in North America, suggesting that these groups had a wider geographic distribution than previously thought; including the Western Tethys Sea or Paleo-gulf of Mexico. The youngest report (Maastrichtian) of Ptychodus mortoni is documented.

Key words: Fish, Mexcala Formation, Upper Cretaceous, Mexico. 


\section{INTRODUCCIÓN}

La presencia de peces fósiles en el Estado de Guerrero, México, solamente había sido documentada hasta el momento por Fries (1960), quien al describir y proponer la Formación Mexcala, vagamente señaló la existencia de escamas en las capas inferiores de esta unidad.

Los restos de peces aquí descritos fueron colectados en rocas del Cretácico Superior (Turoniano-Maastrichtiano) de seis localidades y forman parte de la Formación Mexcala en el Estado de Guerrero (Figuras 1 y 2). Este estudio fue parcialmente reportado por Garibay-Romero \& AlvaradoOrtega (2002) y por Alvarado-Ortega et al. (2006). En este trabajo se describe e ilustra el conjunto total de estos restos fósiles, representando dos grupos mayores de peces: osteictios y condrictios.

Todo el material fósil se encuentra depositado en la Colección de Paleontología de la Unidad Académica de Ciencias de la Tierra, Universidad Autónoma de Guerrero (ERCT).

\section{GEOLOGÍA}

La Formación Mexcala ha sido descrita a partir de afloramientos localizados en el noreste del Estado de Guerrero, el sureste del Estado de Morelos y suroeste del Estado de Puebla, dentro de la Cuenca Morelos-Guerrero (Figura 1). Esta formación, descrita originalmente por Fries (1960), presenta variaciones importantes en sus facies, tanto lateralmente como verticalmente, En general pueden distinguirse dos miembros, uno inferior calcáreo y uno superior terrígeno (GonzálezPacheco, 1988; Hernández-Romano, 1999).

El miembro inferior, también llamado calcáreo, está constituido en algunas localidades por calizas y calizas arcillosas en estratos gruesos a medios, que presentan abundantes bioclástos, intraclástos y en ocasiones son peloidales. Estas varían en textura desde caliza compacta (packstone) a caliza granular (grainstone). Además incluye estratos calcáreos portadores de rudistas, con texturas rudstone y floatstone. Hacia la cima de esta secuencia aparecen estratos de arenisca calcárea, portadores de una abundante fauna bentónica. Las microfacies y el contenido de ostrácodos y miliólidos encontrados en las calizas sugiere que el depósito de este miembro ocurrió en un ambiente marino somero (Fries, 1960; Hernández-Romano, 1999; Aguilera-Franco, 2000).

En el miembro superior, llamado terrígeno, las facies calcáreas cambian transicionalmente a una sucesión de lutitas, lutitas calcáreas, areniscas y conglomerados, estos últimos son más abundantes hacia la cima de la sucesión (sucesión grano-creciente). Esta sucesión clástica contienen esporádicas intercalaciones de calizas. Las estructuras primarias y características texturales sugieren que la mayoría de estos sedimentos fueron transportados por flujos de gravedad (turbiditas, flujos de grano y flujos de detritos).

Las características sedimentológicas y las asociaciones fósiles de la Formación Mexcala sugieren un cambio en los ambientes de depósito que va transicionalmente de un
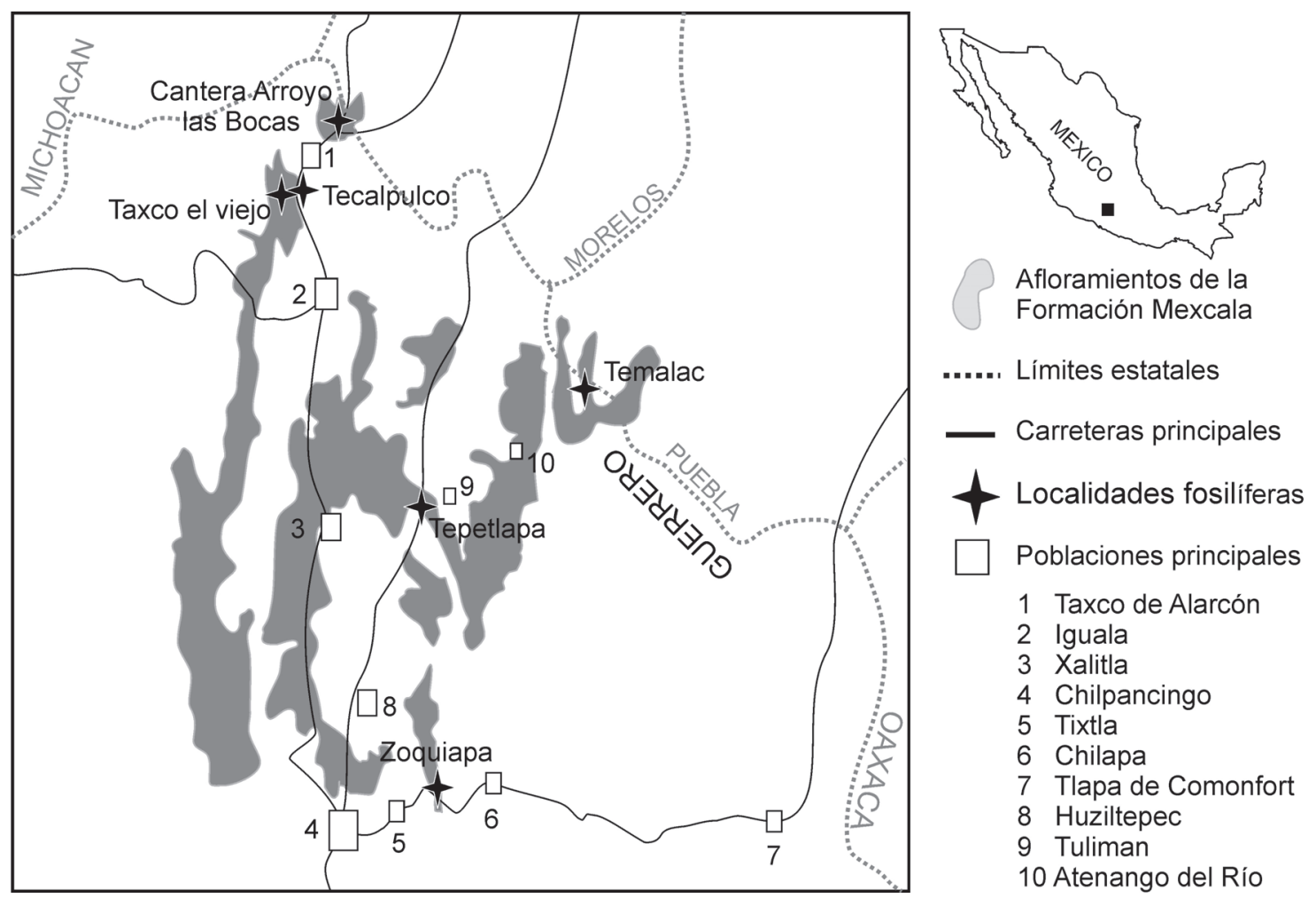

Figura 1. Localidades fosilíferas y distribución geográfica de los afloramientos de la Formación Mexcala (en gris) en el Estado de Guerrero, México.

Figure 1. Fossil localities and distribution of the Mexcala Formation outcrops (gray), in Guerrero State, Mexico. 
ambiente pelágico, a marino somero, a prodelta-planicie deltaica (Aguilera-Franco, 2000).

Los fósiles reconocidos en la Formación Mexcala han sido abordados por varios autores (Burckhardt, 1919; GonzálezArreola, 1977; Alencáster et al., 1987; Vega \& Feldmann, 1992; Perrilliat \& Vega, 1996; Lang \& Frerichs, 1998; Perrilliat et al., 2000; Kiel \& Perrilliat, 2001, 2004, entre otros), quienes han identificado diversos grupos, incluyendo: foraminíferos, corales, bivalvos, gasterópodos, equinoideos, crinoides, escafópodos, cefalópodos, ostrácodos, crustáceos y restos de vertebrados. Los peces fósiles reportados en este trabajo fueron colectados en varios afloramientos de la Formación Mexcala, ubicados en tanto en la región Norte como en la zona Centro del Estado de Guerrero (Figura 1).

\section{Localidades fosilíferas}

Cantera Arroyo Las Bocas. El acceso a esta localidad es por la carretera estatal entre Taxco de Alarcón y las Grutas de Cacahuamilpa (18`38'16”N, 99²8’33”W). Los fósiles fueron colectados en afloramientos expuestos en el Arroyo Las Bocas (Figura 2), ubicado a $500 \mathrm{~m}$ de la desviación hacia Chontalcoatlán-Grutas de Cacahuamilpa. Las rocas que forman el afloramiento son calizas negras finamente laminadas, de textura packestone y wackestone, que contienen foraminíferos planctónicos y radiolarios, asociados con ejemplares identificados como Inoceramus (Mytiloides) labiatus, represéntale cual es un fósil índice para el Turoniano inferior. Taxco el Viejo. Esta localidad se ubica un kilómetro al norte del pueblo Taxco el Viejo (18`29'27’ N, 99³5'00’W). En esta localidad, la Formación Mexcala está constituida por una la alternancia de estratos de lutita, limolita y arenisca calcárea. Los estratos de arenisca presentan gradaciones normales e inversas, estratificación cruzada y laminación paralela, así como estructuras de socavamiento, las cuales sugieren un transporte por corrientes de turbidez. La presencia de Inoceramus (Mytiloides) labiatus permite reconocer que la edad de esta es una localidad corresponde al Turoniano inferior.

Tecalpulco. Esta localidad se encuentra a $300 \mathrm{~m}$ de la salida, hacia el este, del pueblo de Tecalpulco $\left(18^{\circ} 29^{\prime} 30^{\prime \prime} \mathrm{N}\right.$, $99^{\circ} 37^{\prime} 00^{\prime \prime} \mathrm{W}$ ). Las litologías observadas en esta localidad son similares a las descritas para Taxco el Viejo.

Temalac. La localidad se ubica a $5 \mathrm{~km}$ al norte de Temalac, poblado ubicado a $35 \mathrm{~km}$ al SE de Iguala, Guerrero $\left(18^{\circ} 08^{\prime} 21^{\prime \prime} \mathrm{N}, 98^{\circ} 45^{\prime} 10^{\prime \prime} \mathrm{W}\right)$. Los fósiles se colectaron de una sucesión de lutitas, limolitas y areniscas calcáreas, que contiene conglomerados hacia la cima. Estas rocas presentan estructuras primarias, texturas y asociaciones fósiles característicos de ambientes de depósito marinos someros (ver Perrilliat et al., 2000).

Tepetlapa. Esta localidad se encuentra a cuatro $\mathrm{km}$ de la salida noreste del pueblo Atenango del Río $\left(18^{\circ} 06^{\prime} 15^{\prime \prime} \mathrm{N}\right.$, $99^{\circ} 05^{\prime} 15^{\prime}$ 'W). Aquí, la Formación Mexcala esta formada por una alternancia de lutitas y areniscas, que presentan estructuras primarias de depósitos turbidíticos. En esta región, Dávila-Alcocer (1974) y González-Arreola (1977) reportaron la presencia de equinoides y amonitas.
Zoquiapa. Esta localidad se se encuentra en el corte de la carretera

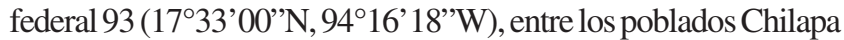
y Zoquiapa, justo en la desviación hacia el poblado El Durazno. En esta localidad también se presenta una alternancia de areniscas y lutitas, y en ella han sido identificados fósiles de diversos bivalvos, gasterópodos y equinoideos.

De acuerdo a Aguilera-Franco (2000), las sucesiones que afloran en las localidades Cantera Arroyo las Bocas, Taxco el Viejo y Tecalpulco pertenecen al miembro profundo o calcáreo de la Formación Mexcala, el cual es interpretado como depositado en las regiones media y externa de una rampa carbonatada de una cuenca marina profunda. En cambio, las facies observadas en Temalac, Tepetlapa y Zoquiapa, sugieren un depósito en un ambiente marino más somero y fueron interpretadas como depósitos de prodelta y planicie deltaica (Aguilera-Franco, 2000).

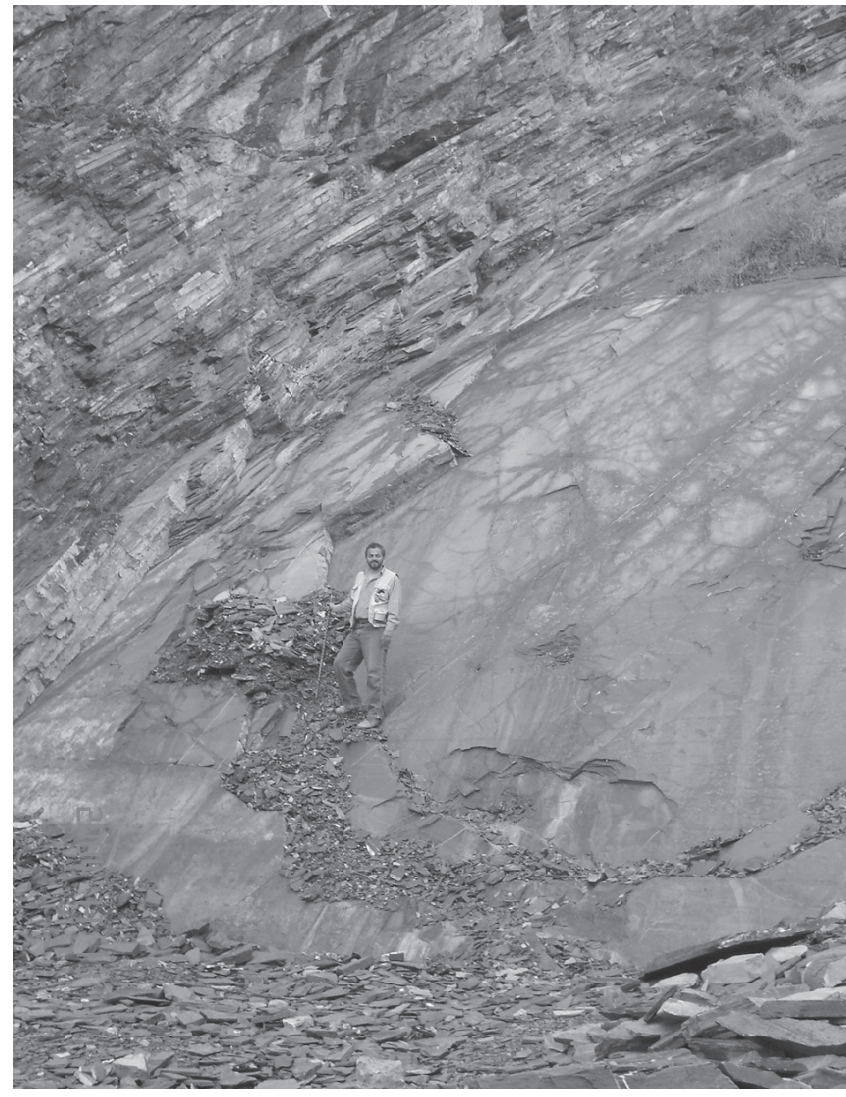

Figura 2. Cantera Arroyo las Bocas, Formación Mexcala, Guerrero. Figure 2. Arroyo las Bocas Quarry, Mexcala Formation, Guerrero.

\section{PALEONTOLOGÍA SISTEMÁTICA}

CHONDRICHTHYES Huxley, 1880

HYBODONTOIDEI Zangerl, 1981 PTYCHODONTIDAE Jaekel, 1898

Ptychodus mortoni Agassiz, 1843

(Figura 3A)

Material. ERCT 2759, diente completo y aislado. Procedencia. Temalac (localidad-IGM 2448). 
Descripción. En vista dorsal, el ejemplar referido es casi cuadrado, con $11 \mathrm{~mm}$ por lado y con el borde anterior derecho (externo) marcadamente redondeado. El área de trituración de la corona muestra una cúspide en forma de domo, en donde hay una serie de crestas distribuidas radialmente desde el centro de la cúspide hasta los bordes marginales.

Comentarios. Ptychodus mortoni ha sido recolectado en sedimentos marinos de Texas, cuya edad comprende el periodo Coniaciano-Santoniano (Williamson et al., 1989; 1990; 1993; Williamson \& Lucas, 1990) y entre el Coniaciano y el Campaniano, distribuidas a lo largo de lo que fuera el Mar Interior de Norteamérica (Applegate, 1970; Cappetta, 1973, 1987; Welton \& Farish, 1993; Cicimurri, 2001; Johnson \& Lucas, 2003, entre otros). En Europa, esta especie ha sido reportada en el rango Cenomaniano-Campaniano (Woodward, 1887, 1889, 1911; Herman, 1977). Recientemente, Antunes \& Cappetta (2002) reconocieron una distribución más amplia de esta especie, la cual durante el Turoniano tardío llegó a estar presente en África.

Ptychodus mortoni ha sido reportado en México, principalmente en localidades de edad turoniana. Los elementos dentales más antiguos de esta especie hasta ahora conocidos (Turoniano temprano) fueron recolectados en Vallecillo, Nuevo León (Blanco-Piñón et al., 2001). Maldonado-Koerdell (1948) y González-Barba et al. (2001), reportaron elementos similares provenientes de sedimentos del Turoniano superior de la Formación Agua Nueva, expuestos en los estados de San Luis Potosí y Coahuila. Anteriormente, Aguilera (1907) reportó esta especie en sedimentos de la misma edad en la localidad Los Peyotes, Coahuila.

El hallazgo de Ptychodus mortoni en los sedimentos de Temalac, permite reconocer una distribución temporal y espacial más amplia para esta especie, al ser éste su primer reporte en sedimentos del Maastrichtiano y su el más austral en Norteamérica.

\section{Ptychodus decurrens Agassiz, 1839}

(Figura 3B)

Material. ERCT 2758, diente aislado completo en matriz rocosa. Procedencia. Taxco el Viejo, Cretácico Superior (Turoniano). Descripción. En vista dorsal, el diente referido tiene forma rectangular, con $12 \mathrm{~mm}$ de ancho y $13 \mathrm{~mm}$ de largo. Su borde anterior es redondeado y el posterior es casi recto. El área de trituración de la corona forma un ligero domo, que presenta una serie de 7 crestas paralelas dispuestas transversalmente. Comentarios. De acuerdo con Cappetta (1987), Ptychodus decurrens fue una especie que durante el Cretácico tardío se desarrolló en las aguas marinas que cubrieron Europa; sin embargo, actualmente se ha reconocido que también estuvo presente en Norteamérica y Australia (Williamson et al., 1990). La distribución temporal de P. decurrens en Norteamérica y Europa comprende el periodo Albiano - Turoniano, mientras que en Australia se ha identificado sólo en el Albiano.

En México, González-Rodríguez \& Applegate (2000) reportaron Ptychodus decurrens en el Albiano-Cenomaniano de la Cantera Muhi, Hidalgo, y González-Barba \& EspinosaChávez (2005) en sedimentos del Cenomaniano-Turoniano de la localidad Jaboncillos, Formación Boquillas, Coahuila. Éste tercer reporte de $P$. decurrens en México, proveniente del miembro profundo de la Formación Mexcala, pudiendo ser su registro más reciente en el país y el más austral en Norteamérica. Por otro lado, este hallazgo permite reconocer que durante el Turoniano, $P$. decurrens tuvo una amplia distribución geográfica en Norteamérica, incluyendo hasta el Estado de Guerrero, México, en lo que entonces fue el Oeste del Mar de Tetis.

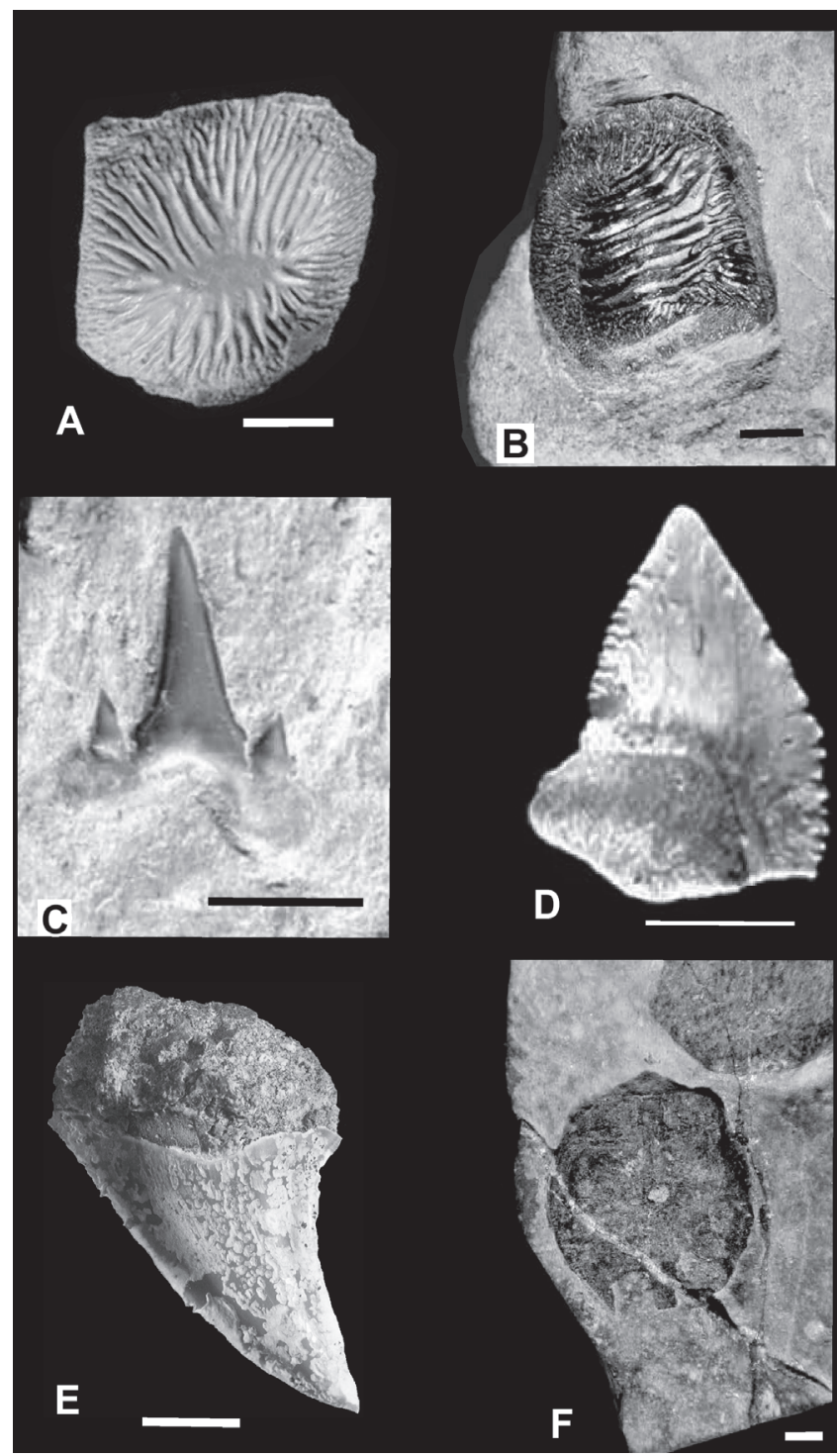

Figura 3. Condrictios de la Formación Mexcala. A, ERCT 2759, diente de Ptychodus mortoni. B, ERCT 2758, diente de Ptychodus decurrens. C, ERCT 2764, diente de Serratolamna serrata. D, ERCT 2760, diente de Squalicorax sp. E, ERCT 2771, corona de un diente de Cretoxyrhina cf. mantelli. F, ERCT 2761, centro vertebral de un lamniforme indeterminado. Escala $=5 \mathrm{~mm}$.

Figure 3. Chondrichthyes from the Mexcala Formation. A, ERCT 2759, tooth of Ptychodus mortoni. B, ERCT 2758, tooth of Ptychodus decurrens. C, ERCT 2764, tooth of Serratolamna serrata. D, ERCT 2760, tooth of Squalicorax sp. E, ERCT 2771, tooth crown of Cretoxyrhina cf. mantelli. F, ERCT 2761, vertebral centrum of an undeterminated lamniform shark. Scale bar $=5 \mathrm{~mm}$. 


\section{LAMNIFORMES \\ SERRATOLAMNIDAE}

\section{Serratolamna serrata (Agassiz, 1843)}

(Figura 3C)

\begin{abstract}
Material. ERCT 2763, diente sin parte del ápice de la corona y sin gran parte de la raíz; ERCT 2764, diente completo.

Procedencia. Tepetlapa, Maastrichtiano temprano.

Descripción. El ejemplar ERCT 2763 muestra la superficie labial. Su forma es triangular casi equilátera. La corona está curvada hacia el extremo posterior. Aunque el extremo apical de la corona no se conservó, es posible considerar que ésta presentaba una altura y anchura (incluyendo los dentículos laterales) de 3,2 y $3,8 \mathrm{~mm}$, respectivamente. Existen dos dentículos laterales en el extremo de la corona, siendo aquél más próximo a la parte central de ésta, tres veces más alto $(0,8 \mathrm{~mm})$ que el colocado en la posición más extrema. En el borde posterior del diente hay solamente un dentículo, cuya altura es ligeramente menor a la del dentículo anterior mayor. Aunque la raíz de este ejemplar sólo está parcialmente conservada, su forma muestra que sus dos lóbulos eran casi simétricos, que estaban separados por una lamela en forma de U invertida, y que sus bordes basales eran planos e igualmente profundos, por lo que casi formaban una línea recta.
\end{abstract}

ERCT 2764, que también expone su superficie labial, tiene una forma más alargada que ERCT 2763 arriba descrito. Esto se debe a que su posición era anterior respecto a la que ERCT 2763 debió ocupar. En el ejemplar ERTC 2764, la corona y la raíz tienen una altura de 4 y $2 \mathrm{~mm}$ respectivamente. La raíz tiene una anchura máxima de 4.4 $\mathrm{mm}$, sus lóbulos posterior y anterior son uniformemente anchos $(1,7 \mathrm{~mm})$, redondeados, y están separados por una lamela profunda, que tiene forma de $\mathrm{U}$ invertida. La profundidad de la raíz en el lóbulo posterior es de 1,1 mm y es ligeramente más alta que el lóbulo anterior. A ambos lados de la corona hay un par de dentículos laterales. Los dentículos laterales mayores son los más próximos a la parte central de la corona, y miden $1 \mathrm{~mm}$ aproximadamente; por el contrario, los dentículos colocados en las posiciones más extremas de la corona son casi cuatro veces menores que los dentículos mayores.

Comentarios. Serratolamna fue erigido para incluir tiburones lamniformes del Cretácico tardío, con dientes caracterizados por la presencia de múltiples dentículos laterales (Landemaine, 1991), como los descritos en los ejemplares aquí referidos.

Existen tres especies incluidas en el género Serratolamna: S. lerichei (Casier, 1946), restringida al Paleógeno de Chesapeake y Carolina del Norte; S. aschersoni (Stromer, 1905), encontrada en sedimentos del Eoceno en las regiones de Chesapeake y el Mississippi (Kent, 1994), y probablemente en Marruecos (Underwood \& Mitchell, 2000); y S. serrata, especie que de acuerdo con Underwood \& Mitchell (2000), es un fósil índice para el Maastrichtiano tardío, ampliamente documentado en Norteamérica, Europa, África y Sudamérica (Wanner, 1902; Rebouças \& Silva-
Santos, 1956; Herman, 1977; Landemaine, 1991; Case \& Cappetta, 1997; Noubhani \& Cappetta, 1997).

En México, Serratolamna serrata también ha sido reportada en sedimentos maastrichtianos de la Formación Cerro del Pueblo, Coahuila (Hernández-Rivera, 1998; Kikland \& Aguillón-Martínez, 2002); en sedimentos de la Cuenca La Popa, pertenecientes a la Formación Potrerillos, del Grupo Difunta, en Nuevo León; así como en sedimentos de la Formación Ocozocuautla, en Chiapas (González-Barba et al., 2001).

Considerando el contexto, la presencia de Serratolamna serrata en Tepetlapa confirma que ésta parte de la Formación Mexcala en Guerrero se depositó durante el Maastrictiano y además, permite reconocer que para ese tiempo esta especie también estuvo ampliamente distribuida en México.

ANACORACIDAE Casier, 1947

Squalicorax sp.

(Figura 3D)

Material. ERCT 2760, diente sin gran parte de la raíz y con la mayor parte de la corona.

Procedencia. Temalac (localidad-IGM 2448), Maastrichtiano temprano.

Descripción. ERCT 2760 conserva prácticamente toda la corona y un fragmento de la raíz. La forma de este diente es triangular, asimétrica y curva hacia la parte distal (posterior); su altura es de $7 \mathrm{~mm}$ y su anchura alcanza los $6 \mathrm{~mm}$ en su base. El borde posterior de este ejemplar es casi recto; mientras que el borde anterior es notoriamente más convexo. Todo el borde de la corona es finamente serrado. Aunque una parte de la región posterior de la raíz está conservada, nada puede señalarse sobre la forma o las dimensiones de esta estructura, salvo que era masiva, proyectada hacia la región lingual.

Comentarios. La distribución de Squalicorax es considerada mundial dentro del rango Albiano-Maastrichtiano (Cappetta, 1987). No es posible llegar a una determinación especifica de ERCT 2760, dada su naturaleza fragmentaria. Sin embargo, la comparación de las características mostradas, tales como la curvatura, la altura y anchura de la corona, permiten reconocer que podría pertenecer a $S$. falcatus (Agassiz, 1843) o a $S$. kaupi (Agassiz, 1843). Estas dos especies son bien conocidas en Norteamérica; $S$. falcatus en un rango temporal Cenomaniano-Campaniano (Welton \& Farish, 1993; Martin et al., 1998; González-Barba \& Espinosa-Chávez, 2005), y $S$. kaupi aparentemente en un rango restringido sólo al Campaniano. Es importante señalar que las diferencias entre ambas especies es ambiguo; en primer lugar, durante la revisión de las características dentarias de estas especies heterodontas Cappetta \& Case (1999) advirtieron que la variación dental de $S$. falcatus incluye formas descritas como S. kaupi; mientras que por otro lado, Martin et al. (1998) señalaron que la morfología de los dientes laterales posteriores en ejemplares pequeños de $S$. kaupi es semejante a la descrita en ejemplares mayores de $S$. falcatus. 


\section{CRETOXYRHINIDAEGlikman, 1958}

\section{Cretoxyrhina cf. mantelli (Agassiz, 1843)} (Figura 3E)

Material. ERCT 2771, diente sin raíz.

Procedencia. Temalac (localidad-IGM 2448), Maastrichtiano temprano.

Descripción. En el ejemplar referido la corona tiene $21 \mathrm{~mm}$ de altura, es marcadamente inclinada posteriormente y su base mide 16,3 mm de ancho. La superficie de la corona es lisa, y su margen cortante es continuo y uniforme hasta la raíz. Hay una amplia banda lingual entre la raíz y la corona. La raíz está parcialmente conservada; sin embargo, por debajo de la corona puede observarse que la raíz era profunda (su profundidad fue al menos la mitad de la altura de la corona), y presentaba una marcada protuberancia lingual.

Comentarios. Cretoxyrhina es un tiburón de África, Europa, Norteamérica, Sudamérica, Oriente Medio y Australia, encontrado en sedimentos depositados dentro del rango Albiano-Campaniano (Welton \& Farish, 1993; Siverson \& Lindgren, 2005).

En Norteamérica Cretoxyrhina, representado por $C$. mantelli, es una forma común en sedimentos depositados en el Mar Interior (Cappetta, 1987; Applegate, 1970; GonzálezBarba \& Espinosa-Chávez, 2005; entre otros). Este tiburón es bien conocido por especimenes en donde gran parte de los dientes se encuentran articulados y asociados (Eastman, 1895; Shimada, 1997). La presencia de Cretoxyrhina en los afloramientos del Maastrichtiano temprano de la Formación Mexcala, en Temalac, Guerrero (Perrilliat et al., 2000), significa que este género logró sobrevivir hasta este periodo, al menos en los mares del extremo sur de Norteamérica, en el extremo oeste del Mar de Tetis.

\section{LAMNIFORMES Indet. \\ (Figura 3F)}

Material. ERCT 2761, vértebra aislada, conservada en matriz rocosa; Cantera Arroyo las Bocas, Taxco. ERCT 2769, vértebra observada en lámina delgada; Zoquiapa, Maastrichthiano temprano.

Descripción. El ejemplar ERCT 2761 representa un centro vertebral aislado, cuyo diámetro varia entre 17 y $19 \mathrm{~mm}$. Como la superficie expuesta está erosionada, es posible observar las cavidades articulares en los bordes dorsal y ventral, cuyas formas cónicas proyectadas al centro permitieron la articulación con los arcos hemal y neural. El ejemplar ERCT 2769 representa un fragmento de un centro vertebral (observado en lámina delgada); donde solo puede observarse parte de las laminillas radiantes formando la estructura interna. En ambos ejemplares la calcificación central presenta un patrón tectospondílico, lo cual es inusual entre los lamniformes.

Comentarios. La inclusión destos ejemplares como lamniformes, se basa en la presencia de laminillas radiantes bien calcificadas, formando la estructura interna de las vértebras, las cuales son características de este grupo de tiburones (e.g. Compagno, 1990; Shirai, 1996; Blanco-Piñón et al., 2005).

\author{
OSTEICHTHYES Huxley, 1880 \\ TELEOSTEI Müller, 1844 \\ ICHTHYODECTIFORMES Bardack \& Sprinkle, 1969 \\ ICHTHYODECTIODEI Indet. \\ (Figuras 4A-B)
}

Material. ERCT 2750, radio pectoral derecho aislado (Figura 4A); ERCT 2751, vértebra aislada; ERCT 2752, escama aislada (Figura 4B).

Procedencia. Cantera Arroyo las Bocas; Cretácico Superior (Turoniano).

Descripción. Es posible reconocer que los ejemplares referidos son restos de peces ictiodectiformes, debido a rasgos característicos y/o diagnósticos de este orden (Bardack, 1965; Patterson \& Rosen, 1977; Maisey, 1991; Taverne \& Chanet, 2000). ERCT 2750 representa uno de los primeros radios de la aleta pectoral derecha, cuya longitud es de al menos $60 \mathrm{~mm}$, mismo que muestra la forma de daga (ancho y plano) diagnóstica de estos peces. Por otro lado, en la vértebra aislada y deformada por compresión, ERCT 2751, aún es posible observar el par de perforaciones laterales encontrado en ictiodectiformes. Finalmente, el ejemplar ERCT 2752 es una escama mostrando una ornamentación superficial con radii anteriores y puntae posteriores; este tipo de escamas son comunes entre ictiodectiformes cretácicos derivados (i.e. Cladocyclus, Vallecillichthys, Ichthyodectes, Gillicus y Xiphactinus).

Comentarios. La presencia de ictiodectiformes en la Cantera Arroyo las Bocas, previamente reportada por Garibay-Romero \& Alvarado-Ortega (2002), extiende la distribución geográfica de este grupo en el país, hasta su parte centro-sur. La edad turoniana de los sedimentos donde éstos fósiles fueron recolectados, está incluida dentro del rango JurásicoCretácico en el que se desarrollaron los ictiodectiformes. Los ictiodectiformes más derivados (incluidos en el Suborden Ichthyodectoidei) aparecieron a partir del Cretácico temprano, y están bien caracterizados por poseer escamas con la misma ornamentación de ERCT 2752, con radii anteriores y puntae posteriores (Alvarado-Ortega, 2005).

$$
\begin{gathered}
\text { TSELFATIIFORMES Nelson, } 1994 \\
\text { TSELFATIIDAE Nelson, } 1994 \\
\text { Tselfatia } \text { sp. } \\
\text { (Figura 4C) }
\end{gathered}
$$

Material. ERCT 2753, fragmento de aleta impar y parte del cuerpo.

Procedencia. Cantera Arroyo las Bocas; Cretácico Superior (Turoniano).

Descripción. La naturaleza fragmentaria del ejemplar referido no permite reconocer si la aleta impar que muestra es la anal o la dorsal. En esta aleta pueden observarse restos de al menos 10 radios, y una sección del cuerpo cubierta por escamas. Ninguno de los radios conservados está completo; 
sin embargo, al parecer hay dos radios notablemente cortos en la parte anterior, y aquél que posiblemente ocupa la tercera posición muestra una composición característica de algunos Tselfatiformes (ver discusión abajo), numerosos pseudofulcros anteriores articulados uno a uno con lepidotriquios colocados en la parte posterior (pueden contarse cerca de 45 de estas estructuras en cerca de $170 \mathrm{~mm}$ de longitud de radio).

Comentarios. De acuerdo con Taverne (2004), entre los Tselfatiiformes sólo los géneros Tselfatia y Dixonanagmius $(=$ Plethodus) presentan un radio distintivo en la aleta dorsal y uno en la anal, el cual es uniformemente ancho y está formado por lepidotriquios y pseudofulcros. La identificación del ejemplar ERC 2753 como parte del primero de estos géneros, está basada en que en Tselfatia, los lepidotriquios y pseudofulcros son igualmente altos, se articulando entre sí en una relación uno a uno. Por el contrario, en Dixonanagmius, los pseudofulcros son notablemente menos altos y varios de éstos se articulan con cada uno de los lepidotriquios (Taverne, 2000).

La familia Tselfatiidae es conocida en sedimentos marinos del Cretácico Superior (Cenomaniano-Santoniano) de África, Europa, Norteamérica y Sudamérica (Páramo-Fonseca, 1997; Fielitz \& Shimada, 1999, entre otros) y incluye Tselfatia formosa Arambourg, 1943; T. dalmatia Bardack \& TellerMarshall, 1980; y posiblemente Bachea huilensis PáramoFonseca, 1997. Aunque la ocurrencia de Tselfatia en los sedimentos cretácicos de Guerrero, previamente señalada por Garibay-Romero \& Alvarado-Ortega (2002), concuerda con la distribución temporal y espacial del grupo, este hallazgo representa el tercer reporte formal del género en México y se suma a aquellos de Vallecillo, Nuevo León y de las canteras al norte de Múzquiz, Coahuila (Blanco-Piñón et al., 2004; Stinnesbeck et al., 2005).

Finalmente, el presente reporte permite reconocer que durante el Turoniano, la distribución de Tselfatia en Norteamérica comprendió hasta el extremo sur de México, al oeste de lo que durante el Mesozoico fue el Mar de Tetis.

\section{AULOPIFORMES Rosen, 1973 DERCETIDAE Indet.}

(Figura 4D)

Material. ERCT 2756, impresión de parte del cráneo en vista lateral; ERCT 2757a/b, parte y contraparte de cráneo y mandíbula desarticulados; ERCT 2765, mandíbula inferior desarticulada.

Procedencia. Cantera Arroyo las Bocas; Cretácico Superior (Turoniano).

Descripción. En todos los ejemplares referidos, sólo se conservan partes de las cabezas donde es evidente el aspecto longirrostrino que estos peces tuvieron. En ERCT 2756 y ERCT 2757, la longitud del cráneo comprendida desde el borde anterior orbital, hasta el borde posterior del opérculo es de 19 y $23 \mathrm{~mm}$, respectivamente. El frontal es muy largo y cubre prácticamente todo el techo del cráneo, incluyendo parte de la región etmoidea. El opérculo es de aspecto rectangular, ligeramente más largo que alto. El preopérculo tiene forma de espátula, y está colocado a lo largo del borde inferior del opérculo. En ERCT 2756 la mandíbula está mal conservada, pero es posible observar que el dentario es corto y que la mandíbula superior se extiende más allá anteriormente. En ERCT 2757, el dentario, el premaxilar, y el maxilar están desarticulados del resto del cráneo. En el borde de alveolar de estos huesos, excepto en el premaxilar, hay una pequeña hilera de finos dientes.

Comentarios. A pesar de que estos fósiles fueron ya referidos como Rhynchodercetis (Alvarado-Ortega et al., 2006), otros dercetidos que comparten características con los ejemplares arriba descritos [Haastaichthys, Taverne 1991; Nardiodercetis Taverne, 2005; Roberthychthys Blanco-Piñón \& Alvarado-Ortega, 2005; y Brazilodercetis Figueiredo \& Gallo, 2006). Material más completo y mejor preservado es necesario para una determinación mejor detallada de estos dercetidos de la Formación Mexcala.

\section{ENCHODONTIDAE Woodward, 1901 Enchodus sp. (Figura 4E)}

Material. ERCT 2754, dentario casi completo aislado. Procedencia. Cantera Arroyo las Bocas, Cretácico Superior (Turoniano).

Descripción. El dentario referido es de forma triangular, su altura es de $10 \mathrm{~mm}$ en la parte posterior y $2 \mathrm{~mm}$ en la sínfisis. El borde ventral también es casi recto y está inclinado respecto al borde alveolar, cerca de 30 grados. Sólo seis dientes rectos con las cúspides agudas y ligeramente curvadas hacia atrás están presentes a lo largo de la parte media y posterior del borde alveolar; mientras que aquellos de la parte anterior no están conservados. La base de los dientes es notablemente ensanchada y está fusionada al dentario. La parte anterior del borde ventral de este hueso presenta tres rebordes salientes (Figura 4E), mismos que han sido identificados como un rasgo distintivo de este género (Goody, 1969).

Comentarios. Enchodus, comúnmente llamado "arenque dientes de sable", fue un pez muy abundante durante el Cretácico tardío (a partir del Cenomaniano), que tuvo una amplia distribución mundial (Goody, 1969; Arambourg, 1952). El hallazgo de esta mandíbula de Enchodus en los sedimentos turonianos explotados en la Cantera las Bocas, se suma a un amplio registro que incluye otras localidades turonianas en México (Alvarado-Ortega et al., 2006). Este género ha sido reportado en San José de las Rusias, Tamaulipas, Xilitla, San Luis Potosí (Maldonado-Koerdell, 1956), Vallecillo, Nuevo León (Blanco-Piñón, 1998), Cantera Muhi, Hidalgo (GonzálezRodríguez \& Applegate, 2000), y recientemente en varias localidades en norte de Coahuila (Blanco-Piñón et al., 2004; González-Barba \& Espinosa-Chávez, 2005). Por el momento, el estudio de los detalles anatómicos de los fósiles de Enchodus encontrados aún no ha sido abordado; sin embargo, el presente trabajo y los arriba citados permiten reconocer que este género está bien representado en el registro fósil del Cretácico Superior de México. 


\section{CROSSOGNATHIFORMES sensu Taverne, 1989 PACHYRHIZODONTIDAE Cope, 1872}

\section{Pachyrhizodus cf. caninus Cope 1872} (Figura 4G)

Material. ERCT 2762, dentario casi completo, desarticulado y aislado; Tepetlapa, Maastrichtiano temprano.

Descripción. ERCT 2762 es un dentario casi completo, cuya forma es rectangular, casi 6 veces más largo que alto. La longitud es de al menos $120 \mathrm{~mm}$ y el proceso coronoideo, cuya altura es de $20 \mathrm{~mm}$, es ligeramente más alto que la sínfisis $(16 \mathrm{~mm})$. Los bordes ventral y alveolar son ligeramente curvos. Sobre el borde alveolar hay una hilera de dientes que contiene 10 dientes fusionados al hueso, cuyos ápices no se conservaron.

La forma del dentario y de los dientes en ERCT 2762 son reconocidos como restos de Pachyrhizodus cf. caninus, dada su semejanza con aquellos restos de esta especie encontrados en sedimentos de Nueva Zelanda y en Norteamérica.

Comentarios. El género Pachyrhizodus incluye numerosas especies y ha sido reconocido en yacimientos cuya edad esta
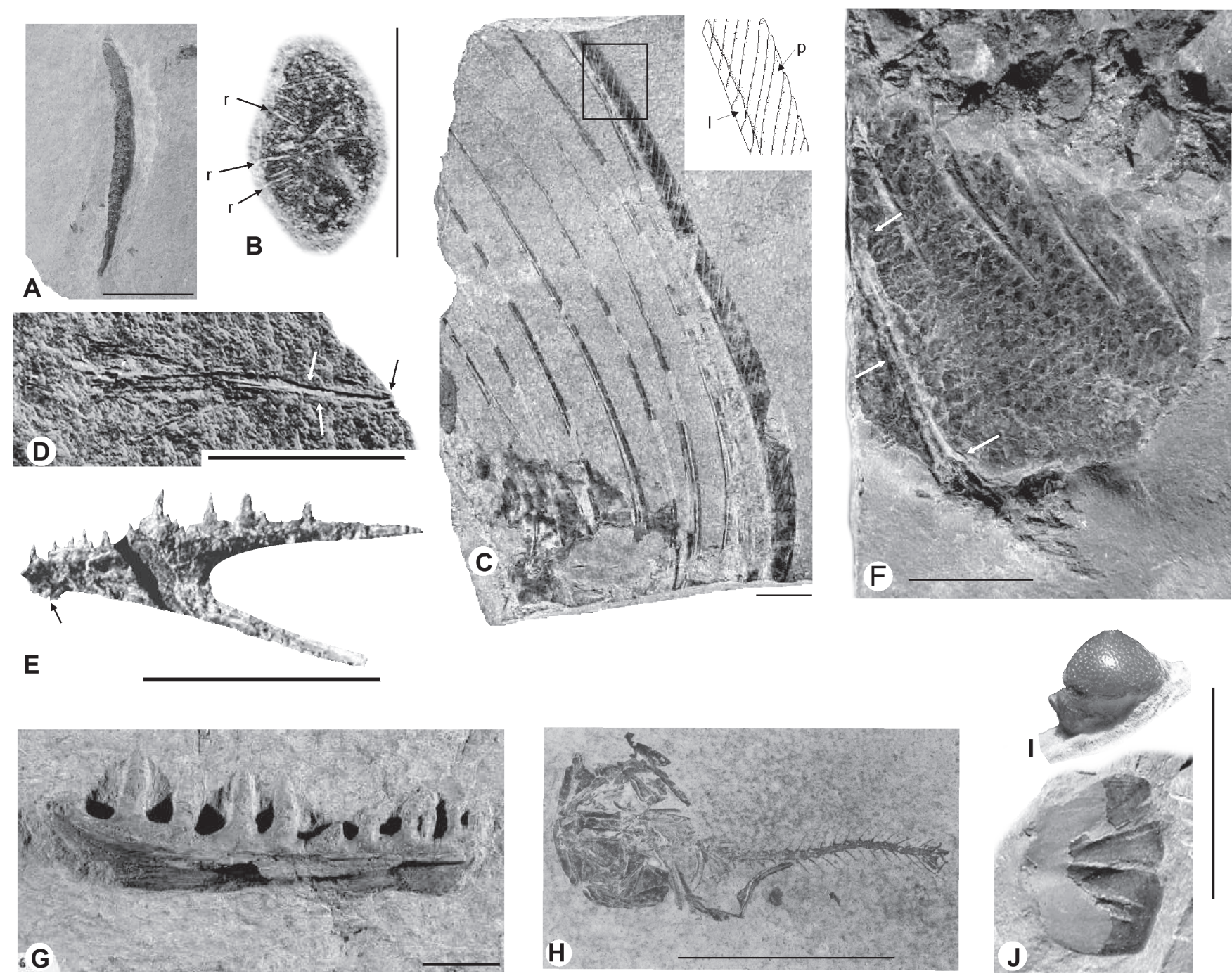

Figura 4. Osteictios de Formación Mexcala. A, ERCT 2750, uno de los primeros radios de la aleta pectoral de un Ichthyodectiformes. B, ERCT 2752, escama de un pez Ichthyodectiformes, mostrando la superficie dorsal ornamentada con radii anteriores (flechas). C, ERCT 2753, fragmento de una aleta impar (posiblemente la dorsal) de Tselfaia sp.; en detalle la reconstrucción de la articulación uno a uno entre los lepidotriquios (I) y pseudofulcros (p). D, ERCT 2757a, parte anterior del cráneo de un Dercetidae, las flechas indican el premaxilar. E, ERCT 2754, dentario de Enchodus sp., la flecha señala el reborde saliente en la región ventral anterior. F, ERCT 2766a, pez teleósteo, las flechas indican el largo proceso anterior del hueso pélvico. G, ERCT 2762, dentario de Pachyrhizodus cf. caninus. H, ERCT 2755, teleosteo. I, ERCT 2768, diente de pez teleosteo. J, ERCT 2767, escama de pez óseo. Escala = $20 \mathrm{~mm}$.

Figure 4. Osteichthyans from the Mexcala Formation. A, ERCT 2750, one of the most anterior pectoral-fin ray of an ichthyodectiform. B, ERCT 2752, scale of an ichthyodectiform showing the superficial anterior radii (arrows). C, ERCT 2753, fragment of an unpaired fin of Tselfaia sp.; reconstruction showing the relation between lepidotrichia (I) and pseudofulcra (p) in detail. D, ERCT 2757a, anterior part of the skull of an Dercetidae.; arrows indicate the premaxilla. E, ERCT 2754, dentary bone of a Enchodus sp.; arrow indicates its anteroventral projection anterior of this bone. F, ERCT 2766a, teleost fish, showing the anterior process of the pelvic bone (arrows). G, ERCT 2762, dentary of Pachyrhizodus cf. caninus. H, ERCT 2755, a teleost. I, ERCT 2768, tooth of a teleost fish. J, ERCT 2767, osteichthyan fish scale. Scale bar $=20 \mathrm{~mm}$. 
incluida en rango Albiano-Maastrichtiano en África, Australia, Nueva Zelanda, Europa, Medio Oriente, Norteamérica y Sudamérica (Bardack, 1965; Goody, 1969; Bartholomai, 1969; Applegate, 1970; Wiffen, 1983; entre otros).

El ejemplar ERCT 2762 fue encontrado aislado en el mismo nivel estratigráfico que los dientes de Serratolamna serrata (ERCT 2763 y ERCT 2764; Figura 3C), lo cual permite confirmar la presencia de Pachyrhizodus en los sedimentos del Maastrichtiano de la Formación Mexcala.

TELEOSTEI Indet.

(Figuras 4F, H-J)

Material. ERCT 2755, ejemplar casi completo sin escamas conservadas (Figura 4H); Cantera Arroyo las Bocas. ERCT 2766a/b, parte y contraparte de la región media del tronco incluyendo la cintura pélvica (Figura 4F); Cantera Arroyo las Bocas. ERCT 2768, diente aislado (Figura 4I); Temalac. ERCT 2767, escama aislada (Figura 4J); Temalac. ERCT 2770, fragmento de columna vertebral; Tecalpulco.

Comentarios. La naturaleza fragmentaria de estos ejemplares no permite observar detalles que permitan su identificación taxonómica. Aunque el ejemplar ERCT 2755 es un pez está casi completo, en este no ha sido posible observar detalles anatómicos que permitan su asignación taxonómica más precisa. Por otro lado, en ERCT 2766, la presencia de un hueso pélvico con un proceso anterior muy desarrollado, cuya longitud es cercana a aquella de cuatro centros vertebrales abdominales (Figura 4F), fue descrita por Cavin (1995) como un rasgo distintivo de Goulmimichthys. Este género

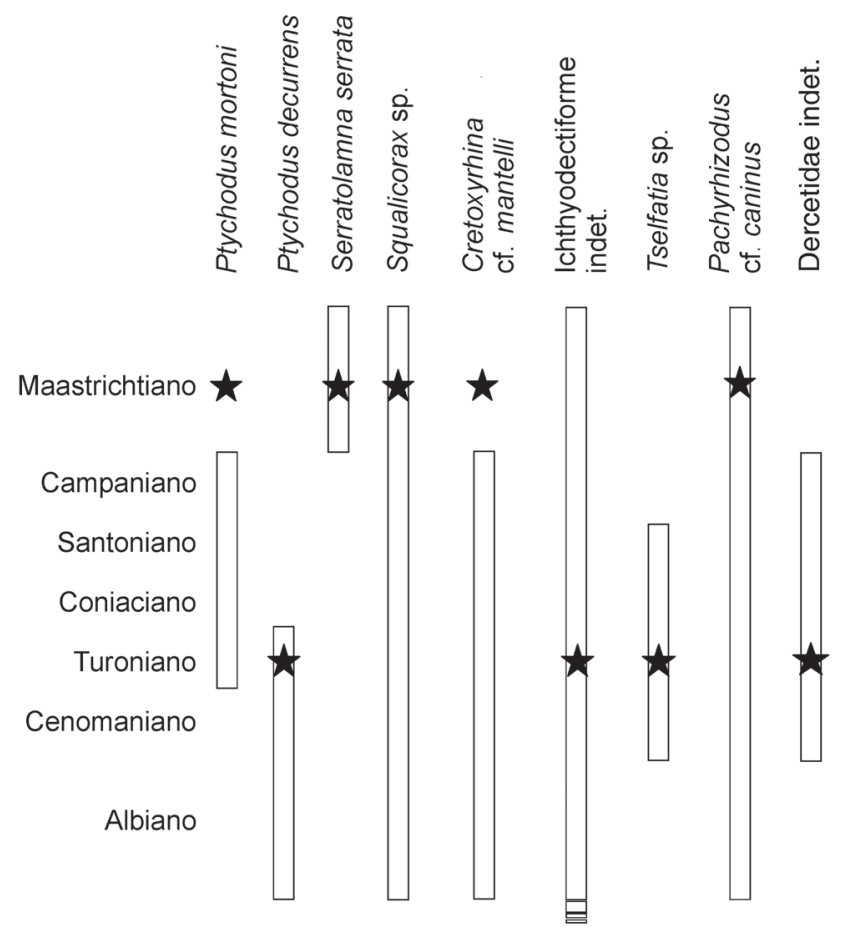

Figura 5. Distribución de los taxones de peces fósiles identificados en la Formación Mexcala, Guerrero, México.

Figure 5. Geological range of the fish taxa found in the Mexcala Formation, Guerrero, Mexico. paquirizodóntido fue descubierto originalmente en los sedimentos marinos del Turoniano en Marruecos, y posteriormente reportado en localidades de la misma edad en Colombia (Páramo-Fonseca, 1997) y Vallecillo, al norte de México (Blanco-Piñón \& Cavin, 2003). ERCT 2767 é similar a las escamas reportadas por Fries (1960).

\section{CONCLUSIONES}

Este trabajo constituye la primera descripción de los peces fósiles en el Estado de Guerrero. Éstos fósiles representan la asociación de peces más austral de Norteamérica documentada hasta el momento, ya que los restos de peces fósiles del Cretácico Superior colectados en Ocozocuautla de Espinosa, Chiapas, no han sido estudiados con suficiente detalle. Adicionalmente, en este trabajo se reporta el hallazgo más reciente (Maastrichtiano) de Ptychosdus mortoni, y posiblemente de Cretoxyrhina mantelli, especies que anteriormente sólo eran conocidas hasta el Campaniano (Figura 5). A pesar de que los rangos de distribución temporal del resto de los peces descritos concuerdan con aquellos previamente conocidos para cada grupo, la distribución paleogeográfica conocida de prácticamente todos ellos se extiende, ya que la asociación de peces fósiles de la Formación Mexcala es afín a otras faunas del Cretácico Superior descritas en lo que fuera el Mar Interior de Norteamérica y el Mar de Tetis (que incluye regiones de Sudamérica, África, Europa y Medio Oriente). En el caso de Rhynchodercetis, Tselfatia y Pachyrhizodus, se consideraban como taxa con distribuciones geográficas limitadas, unos al Mar Interior de Norteamérica, y otos a los extremos sur y este del mar de Tetis (incluyendo Norte de África y Sudamérica; así como Medio Oriente y Europa). El encontrarse éstos organismos en el sur de México, que para el Cretácico Tardío ya formaba parte de la región sur de Norteamérica, sugiere una distribución más amplia y continua para estos peces fósiles, lo cual es un descubrimiento importante para el modelado de la distribución geográfica de los mares del Cretácico.

\section{AGRADECIMIENTOS}

Este trabajo fue posible gracias al apoyo de L. MartínMedrano, G. Álvarez-Reyes y J.M. Padilla-González, quienes asistieron a los autores en los trabajos de campo. A L. Espinosa-Arrubarrena le agradecemos las sugerencias brindadas a las primeras versiones de este trabajo. Todo el material fotográfico fue elaborado por Antonio Altamira. El trabajo de campo fue apoyado por el Departamento de Paleontología del Instituto de Geología, UNAM. JAO agradece al Conselho Nacional de Desenvolvimento Cientifico e Tecnológico el soporte financiero (proc. 151115/ 2005-2).

\section{REFERENCIAS}

Agassiz, J.L.R. 1835-1843. Recherches sur les poisons fossils, Neuchâtel, Universitat Neuchâtel, 1420 p. 
Aguilera, J.G. 1907. Apercu sur la Géologie du mexique pour servir d'explication á la carte géologique de l'Amérique du Nord. In: CONGRÉS GÉOLOGIQUE INTERNATIONAL, 1, 1906. Compte Rendu de la Dexieme Session, Paris, p. 227-248.

Aguilera-Franco, N. 2000. High resolution stratigraphy and palaeoecology of the Cenomanian-Turonian succession, Southern México. T.H. Huxley School, University of London, Tesis de Doctorado, $244 \mathrm{p}$.

Alencáster, G.; Hernández-García, R. \& García-Villegas, F. 1987. Rudistas hipuritidos (Bivalvia-Hippuritacea) del Cretácico superior de la parte central del Estado de Guerrero. Revista de la Sociedad Mexicana de Paleontología, 1:24-39.

Alvarado-Ortega, J. 2005. Sistemática de los peces Ichthyodectiformes de la Cantera Tlayúa, Puebla, México. Posgrado en Ciencias Biológicas, Universidad Nacional Autónoma de México, Tesis de Doctorado, 308 p.

Alvarado-Ortega, J; González-Rodríguez, K.A; Blanco-Piñón, A.; Espinosa-Arrubarrena, L. \& Ovalles-Damián, E. 2006. Mesozoic Osteichthyans of Mexico. In: F.J. Vega, T.G. Nyborg, M.C. Perillita, M. Montellano-Ballesteros, S.R.S. CevallosFerriz \& S.A. Quiroz-Barroso (eds.) Studies on Mexican Paleontology, Springer, p. 169-207.

Antunes, M.T. \& Cappetta, H. 2002. Sélaciens de Crétace (AlbienMaastrichtien) d' Angola. Palaeontographica, Abteilung, A, 264:85-146.

Applegate, S.P. 1970. The vertebrate fauna of the Salma Formation of Alabama, Part VIII, The Fishes. Fieldiana Geology Memoirs, 3(8):383-433.

Arambourg, C. 1943. Note préliminaire sur quelques poissons fossiles nouveaux. Bulletin de la Société Géologique de France, 5(8):281-288.

Arambourg, C. 1952. Révision des poissons fossiles de Licata (Sicile). Annales Paleontologiques, 14:39-132.

Bardack, D. 1965. Anatomy and evolution of Chirocentrid fishes. The University of Kansas Paleontological Contributions, 10:1-88.

Bardack, D. \& Teller-Marshall, S. 1980. Tselfatia, a Tethyan Cretaceous Teleost, first records from North America and Yugoslavia. Journal of Paleontology, 54(5):1075-1083.

Bartholomai, A. 1969. The Lower Cretaceous elopid fish Pachyrhizodus marathonensis (Etheridge Jr.). In: K.S.W. Campbell (ed.) Stratigraphy and Paleontology, Australian National University Press, p. 249-263.

Blanco-Piñón, A. 1998, Vallecillo, Nuevo León: yacimiento fosilífero del Noreste de México. Programa de posgrado, Universidad Autónoma de Nuevo León, Tesis de Maestría, 148 p.

Blanco-Piñón, A. \& Alvarado-Ortega, J. 2005. New dercetid fish (Aulopiformes: Teleostei) from the Early Turonian of Vallecillo, NE México. In: INTERNACIONAL MEETING ON MESOZOIC FISHES -SYSTEMATICS, HOMOLOGY, AND NOMENCLATURE, 4, 2005. Extended Abstracts, UNAM Ediciones, p. 43-46.

Blanco-Piñón, A. \& Cavin, L. 2003. New Teleostei from the Agua Nueva Formation (Turonian), Vallecillo (NE Mexico). Comptes Rendus Paleovol, 2:299-306.

Blanco-Piñón, A.; Porras-Múzquiz, H., Vega, F.; González-Rodríguez, K. A. \& Alvarado-Ortega, J. 2004. Múzquiz, Coahuila: a new fossiliferous locality, northern Mexico: In: CONGRESO NACIONAL DE PALEONTOLOGÍA, 9, 2004. Libro de resúmenes, Sociedad Mexicana de Paleontología, Chiapas, p. 23.

Blanco-Piñón, A.; Shimada, K. \& González-Barba, G. 2005. Lamnoid vertebrae from Agua Nueva Formation (Upper Cretaceous: lower Turonian), northeastern México. Revista Mexicana de Ciencias Geológicas, 22(1):19-23.

Blanco-Piñón, A.; Stinnesbeck, W.; López-Oliva, J.G.; Frey, E.; Adatte, T. \& González, A. H. 2001. Vallecillo, Nuevo León: una nueva localidad fosilífera del Cretácico tardío en el noreste de México. Revista Mexicana de Ciencias Geológicas, 18(2):186-199.

Burckhardt, C. 1919. Faunas jurásicas de Simón (Zacatecas) y faunas cretácicas de Zumpango del Río (Guerrero). Buletin del Instituto Geológico de México, 136 p. (Boletín 33)

Cappetta, H. 1973. Selachians from the Carlile Shale (Turonian) of South Dakota. Journal of Paleontology, 47(3):504-514.

Cappetta, H., 1987. Chondrichthyes II-Mesozoic and Cenozoic Elasmobranchii. Stuttgart, Gustav Fischer Verlag, 193 p.

Cappetta, H. \& Case, G.R. 1999. Additions aux faunes des sélaciens du Crétace du Texas (Albien-Campanien). Palaeo Ichthyologica, 9:1-142.

Case, G.R. \& Cappetta, H. 1997. A new selachian fauna from the Late Maastrichtian of Texas (Upper Cretaceous/Navarroan; Kemp Formation). Münchner Geowissenschaftliche Abhandlungen (A: Geologie und Paläontologie), 34:131-189.

Casier, E. 1946. La faune ichtyhyologique de'l'Yprésien de la Belgique. Mémories du Musee Royal d'Histoire Naturelle de Beligique, 104:1-267.

Cavin, L. 1995. Goulmimichthys arambourgi n. g., n. sp., un Pachyrhizodontidae (Actinopterygii, Teleostei) d'une nouvelle localité nodules fossilifres du Turonien inférieur marocain. Comptes Rendus de l'Académie des Sciences, 321:1049-1054.

Cicimurri, D. 2001. Cretaceous elasmobranchs of the Greenhorn Formation (Middle Cenomanian-Middle Turonian), western South Dakota: In: V. L. Santucci \& L. McClelland (eds.) Proceedings of the Sixth Fossil Resource Conference, Geologic Resources Division Technical Report, p. 27-43.

Compagno, L.J.V. 1990. Relationships of the megamouth shark, Megachasma pelagios (Lamniformes, Megachasmidae), with comments on its feeding habits: In: H. L. Pratt Jr., S.H. Gruber \& T. Taniuchi (eds.) Elasmobranchs as living resources, advances in the biology, ecology, systematics, and status of the fisheries, Technical Report 90, U.S. Natural Marine Fish Service, p. 357-380.

Cope, E.D. 1872. On the families of the Cretaceous formations in Kansas. Procedings of American Philosophical Society, Philadelphia, 12:327-357.

Dávila-Alcocer, V.M. 1974, Geología del área de Atenango del Río, Estado de Guerrero. Universidad Nacional Autónoma de México, Tesis de Licenciatura, 109 p.

Eastman, C. 1895. Beiträge zur Kenntnis der Gattung Oxyrhina, mit besonderer Berücksichtigung von Oxyrhina mantelli Agassiz. Palaeontographica, 41:149-192.

Fielitz, C. \& Shimada, K. 1999. A new species of Bananogmius (Teleostei: Tselfatiiformes) from the Upper Cretaceous Carlile Shale of Western Kansas. Journal of Paleontology, 73(3):504-511.

Figueiredo, F.J. \& Gallo, V. 2006. A new dercetid fish (Neoteleostei: Aulopiformes) from the Turonian of the Pelotas Basin, southern Brazil. Palaeontology, 49(2):445-456.

Fries Jr., C. 1960. Geología del Estado de Morelos y partes adyacentes de México y Guerrero, región central meridional de México. Instituto de Geología, 236 p. (Boletín 60)

Garibay-Romero, L.M. \& Alvarado-Ortega, J. 2002. El hallazgo de peces fósiles en el Arroyo "Las Bocas" y la Cantera "Temazol", Cretácico Superior, en la región norte del Estado de Guerrero. In: CONGRESO NACIONAL DE PALEONTOLOGÍA, 8, 
2002. Libro de resúmenes, Sociedad Mexicana de Paleontología, Guadalajara, p. 104.

González-Arreola, C. 1977. Amonitas del Coniaciano (Cretácico Superior) de la región de Tepetlapa, Estado de Guerrero. Revista del Instituto de Geología, 1(2):167-173.

González-Barba, G.; Coutiño José, M.A.; Ovalles Damian, E. \& Vega, F. J. 2001. New Maastrichtian elasmobrach faunas from Baja California Peninsula, Nuevo León and Chiapas states, México. In: INTERNATIONAL MEETING ON MESOZOIC FISHES, 3, 2001. Abstract Book, Sepiano, p. 33.

González-Barba, G. \& Espinosa-Chavez, B. 2005. CenomanianTuronian fish fauna from the Boquillas Formation, North-West Coahuila, Mexico. In: INTERNATIONAL MEETING ON MESOZOIC FISHES, 4, 2005. Extended Abstracts, UAM Ediciones, Madrid, p. 105-108.

González-Pacheco, V. 1988. Estratigrafía y condiciones paleoambientales de la Secuencia Cretácica del área de Atenango del Río, Estado de Guerrero. Universidad Nacional Autónoma de México, Tesis de Licenciatura, 102 p.

González-Rodríguez, K. \& Applegate, S.P. 2000. Muhi Quarry, a new Cretaceous fish locality in Central Mexico. Journal of Vertebrate Paleontology, 20(supl. 3):45A.

Goody, P.C. 1969. The relationships of certain Upper Cretaceous Teleosts with special reference to the Myctophids. Bulletin of the British Museum (Natural History), 7:1-255.

Herman, J. 1977. Les sélaciens des terrains néocrétacés et paléocenes de Belgique et des contrées limitrophes. Eléments d'une biostratigraphique inter-continentale. Mémoires pour Sérvir a l'Explication des Cartes Géologiques et Miniéres de la Belgique. 15:1-401.

Hernández-Rivera, R. 1998. Las playas del Cretácico en Coahuila: In: CONGRESO NACIONAL DE PALEONTOLOGÍA, 4, 1998. Libro de Resúmenes, Sociedad Mexicana de Paleontología, Ciudad de México, p. 33.

Hernández-Romano, U. 1999. Facies, stratigraphy, and diagenesis of the Cenomanian-Turonian of the Guerrero-Morelos Platform, southern México. Postgraduate Research Institute for Sedimentology, University of Reading, Tesis de Doctorado, $322 \mathrm{p}$.

Johnson, S.C. \& Lucas, S.G. 2003. Selachian fauna from the Upper Cretaceous Dalton Sandstone, middle Rio Puerco Valley, New Mexico. FIELD GEOLOGY OF THE ZUNI PLATEAU CONFERENCE, 54, 2003. Guidebook, New Mexico Geological Society, p. 353-358.

Kent, B.W. 1994. Fossil Sharks of the Chesapeake Region. Maryland, Egan Rees \& Boyer, 146 p.

Kiel, S. \& Perrilliat, M.C. 2001. New gastropods from the Maastrichtian of the Mexcala Formation in Guerrero, southern Mexico, part I: Stromboidea. Neues Jarhbuch für Geologie und Paläontologie, Abhandlung, 222:407-426.

Kiel, S. \& Perrilliat, M.C. 2004. New gastropods from the Maastrichtian of the Mexcala Formation in Guerrero, southern Mexico, part III: higher Caenogastropoda. Neues Jarhbuch für Geologie und Paläontologie, Abhandlung, 231:191-217.

Kirkland, J.I. \& Aguillón-Martínez, M.C. 2002. Schizorhiza: a unique sawfish paradigm from the Difunta Group, Coahuila, Mexico. Revista Mexicana de Ciencias Geológicas, 19(1):1624.

Landemaine, O. 1991. Selaciens nouveaux du Cretace Superieur du Sud-Oust de la France, quelques apports a la systematique des elasmobranches. Société Amicale des Géologues Amateures, 1:1-45.
Lang, H.R.\& Frerichs, W.E. 1998. New planktic foraminiferal data documenting Coniacian age for Laramide Orogeny and paleooceanography in Southern Mexico. The Journal of Geology, 106:635-640.

Maisey, J.G. 1991. Santana Fossils: an illustrated atlas. New Jersey, T.F.H. Publications Inc, 459 p.

Maldonado-Koerdell, M. 1948. Peces fósiles de México, I. Elasmobranquios. Revista de la Sociedad Mexicana de Historia Natural, 9:127-133.

Maldonado-Koerdell, M. 1956. Peces fósiles de México III, Nota preliminar sobre peces del Turoniano superior de Xilitla, San Luis Potosí, México. Ciencia (México), 16:31-36.

Martin, J.E.; Schumacher, B.A.; Parris, D.C. \& Grandstaff, B.S. 1998. Fossil vertebrates of the Niobrara Formation in South Dakota: In: J.E. Martin; J.W. Hoganson \& R.C. Benton R.C. (eds.) Proceedings for the Fifth Conference on Fossil Resources, Dakoterra, p. 39-54.

Nelson, J.S. 1994. Fishes of the world. New York, USA, John Wiley \& Sons, 600 p.

Noubhani, A. \& Cappetta, H. 1997. Les Orectolobiformes, Carcharhiniformes et Myliobatiformes (Elasmobranchii, Neoselachii) des bassins à phosphate du Maroc (MaastrichtienLutétien basal); Systématique, biostratigraphie, évolution et dynamique des faunes. Palaeo Ichthyologica, 8:1-327.

Páramo-Fonseca, M. 1997. Bachea huilensis nov.gen., nov. sp., premier Tselfatoidei (Teleostei) de Colombia. Academia des Sciences, 325:147-150.

Patterson, C. \& Rosen, D.E. 1977. Review of ichthyodectiform and other Mesozoic teleost fishes and the theory and practice of classifying fossils. Bulletin of the American Museum of $\mathrm{Na}$ tural History, 158:81-172.

Perrilliat, M.C. \& Vega, F.J. 1996. A new species of Calyptraphorus (Mesogastropoda, Strombidae) from the Maastrichtian of southtern Mexico; some paleobiogeographic and evolutionary implications. Tulane Studies in Geology and Paleontology, 29(34):119-128.

Perrilliat, M.C.; Vega, F.J. \& Corona, R. 2000. Early Maastrichtian Mollusca from the Mexcala Formation of the State of Guerrero, southern Mexico. Journal of Paleontology, 74(1):7-24.

Rebouças, J.C. \& Silva-Santos, R. da, 1956. Fauna ictiologica do fosfato de Pernambuco. Boletim da Divisão de Geologia e Mineralogia, 162:1-29.

Shimada, K. 1997. Skeletal anatomy of the Late Cretaceous lamniform shark, Cretoxyrhina mantelli, from the Niobrara Chalk in Kansas. Journal of Vertebrate Paleontology, 17(4):642-652.

Shirai, S. 1996. Phylogenetic interrelationships of neoselachians (Chondrichthye: Euselachii): In: M.L.J. Stiassny, L.R. Parenti \& G.D. Jonson (eds.) Interrelationships of Fishes, Academic Press, p. 9-34.

Siverson, M. \& Lindgren, J. 2005. Late Cretaceous sharks Cretoxyrhina and Cardabiodon from Montana, USA. Acta Palaeontologica Polonica, 50(2):301-314.

Stinnesbeck, W.; Ifrim, C.; Schmidt, H.; Rindfeisch, A.; Buchy, M.C.; Frey, E.; González-González, A.; Vega, F.J.; Cavin, L.; Keller, G. \& Smith, K.T. 2005. A new lithographic limestone deposit in the Upper Cretaceous Austin Group at El Rosario, County of Múzquiz, Coahuila, northeastern Mexico. Revista Mexicana de Ciencias Geológicas, 22(3):401-418.

Stromer, E. 1905. Die fischreste des mittleren und oberen Eocäns von Ägypten. I. Teil: Selachii, B. Squaloidei, und II. Teil: Teleostomi, A. Ganoidei. Beitrage zur Paläontologie und Geologie, 18:163-192. 
Taverne, L. 1991. New considerations on the osteology and phylogeny of the Cretaceous marine teleost family Dercetidae. Biologisch Jaarboek Dodonae, 58:94-112.

Taverne, L. 2000. Tselfatia formosa, téleostéen marin du Crétacé (Pisce, Actinopterygii), et la position systématique des Tselfatiifromes ou Bananogmiiformes. Geodiversitas, 22(1):5-22.

Taverne, L. 2004. Ostéologie de Pentanogmius evolutus_(Cope, 1877) n. comb. (Teleostei, Tselfatiiformes) du Crétacé supérieur marin des États-Unis-Remarques sur la systématique du genre Pentanogmius Taverne, 2000. Geodiversitas, 26(1):89-113.

Taverne, L. 2005. Les poisons crétacés de Nardò. $22^{\circ}$ Nardodercetis vandewallei gen. et sp. nov. (Telesotei, Aulopiformes, Dercetidae). Bollettino del Museo Civico di Storia Naturale di Verona, Paleontologia, 29:39-54.

Taverne, L. \& Chanet, B. 2000. Faugichthys loryi n. gen., n. sp. (Teleostei, Ichthyodectiformes) de l'Albien terminal (Crétacé inférieur marin) du vallon de la Fauge (Isère, France) et considérations sur la phylogénie de Ichthyodectidae. Geodiversitas, 22(1):23-34.

Underwood, C.J. \& Mitchell, S. 2000. Serratolamna serrata (Agassiz) (Pisces, Neoselachii) from the Maastrichtian (Late Cretaceous) of Jamaica. West Indian Journal of Earth Sciences, 34:25-30.

Vega, F.J. \& Feldmann, R.M., 1992, Occurrence of Costacopluma (Decapoda Brachyura Retroplumidae) in the Maastrichtian of southern Mexico and its paleogeographic implications. Annals of Carnegie Museum, 61(2):133-152.

Wanner, J. 1902. Die fauna der obersten weissen Kreide der libyschen Wüste. Palaeontographica, 30(2):91-152.

Welton, B.J. \& Farish, R.F. 1993. The Collectors Guide to Fossil Sharks and Rays from the Cretaceous of Texas. Dallas, USA, Horton Printing Company, 204 p.
Wiffen, J. 1983. The first record of Pachyrhizodus caninus Cope (Order Clupeiformes) from the Late Cretaceous of New Zealand. New Zealand Journal of Geology and Geophysics, 26:109-119.

Williamson, T.E.; Kirkland, J.I. \& Lucas, S.G. 1993. Selachians from the Greenhorn ciclothem ("Middle" Cretaceous; Cenomanian-Turonian), Black Mesa, Arizona, and the paleogeographic distribution of Late Cretaceous Selachians. Journal of Paleontology, 67:447-474.

Williamson, T.E. \& Lucas, S.G. 1990. Late Cretaceous vertebrates from the Mulatto Tongue of the Mancos Shale, central New Mexico. New Mexico Journal of Science, 30:27-34.

Williamson, T.E.; Lucas, S.G. \& Kirkland, J.I. 1990. The Cretaceous elasmobranch Ptychodus decurrens Agassiz from North America. Geobios, 24(5):595-599.

Williamson, T.E.; Lucas, S.G. \& Pence, R. 1989. Selachians from the Hosta Tongue of Point Lookout Sandstone (Upper Cretaceous, Santonian) central New Mexico. New Mexico Geological Society, Guidebook, 40:239-245.

Wilson, M.V.H. \& Chalifa, Y. 1989. Fossil marine actenopterigyan fishes from the Kaskapau Formation (Upper Cretaceous: Turonian) near Watino, Alberta. Canadian Journal of Earth Sciences, 26:2604-2620.

Woodward, A.S. 1887. On the dentition and affinities of the selachian genus Ptychodus Agassiz. Quarterly Journal of the Geological Society, 43:121-131.

Woodward, A.S. 1889. Catalogue of the fossil fishes in the British Museum (Natural History) Part I, Elasmobranchii: London, British Museum, $474 \mathrm{p}$.

Woodward, A.S. 1911. The fossil fishes of the English Chalk. Palaeontology Society, 64(6):185-224.

Received in May, 2006; accepted in September, 2006 